\title{
La formación universitaria de periodistas en México
}

\author{
María Elena HernándeZ*
}

El propósito de este artículo es reconstruir la trayectoria de la enseñanza del periodismo en México, como un antecedente necesario para discutir el tema de la llamada "profesionalización" de este oficio. Mediante la descripción de los grandes problemas reconocidos incluso por las propias universidades, se quiere señalar la falta de una definición de la sociedad mexicana frente al periodismo, a las funciones que debería cumplir, y al perfil deseable del periodista formado en la universidad. $\mathrm{Si}$ bien el problema de la calidad del periodismo no se reduce a la calidad de su enseñanza, a poco más de medio siglo del establecimiento del primer programa universitario para formar periodistas, permanece el cuestionamiento sobre el papel que ejercen estas escuelas en el mejoramiento de la calidad del periodismo.
This article aims to rebuild the course of journalism education in México, as a necessary step for the discussion of the so called professionalisation of journalism. By describing the main problems of J-schools, it is suggested that Mexican society lacks of stance about the kind of professional universities should make. It is been more than 50 years since the first J-school was founded, and the question regarding the role of these institutions in the improving of Journalism remains. 
Cuando en México se discute sobre la calidad del periodismo, existe una marcada tendencia a calificar de "poco profesional" al mal periodismo, y de "profesional" al que se considera satisfactorio. Esto lleva, casi inevitablemente, al deseo o exigencia de que se "profesionalicen" las prácticas periodísticas, sin que se tenga en verdad claro en qué se piensa cuando se pide esa "profesionalización". ${ }^{1}$

Una indagación académica al respecto obliga, entre otros caminos, a pensar en la sociología de las profesiones como una posible entrada para ubicar el estatus profesional del oficio periodístico. Aunque, al internarnos en las múltiples corrientes de esta sociología, la cuestión se vuelve más confusa o compleja que al inicio.

De acuerdo con los cánones de las profesiones liberales, en su esquema más clásico (y aquí simplificado), un oficio puede considerarse "profesionalizado" cuando cuenta con: un saber específico, un código ético que regule las prácticas profesionales y una organización gremial sólida, o colegiación de los practicantes.

En teoría, podría entenderse que la presencia de escuelas de periodismo muestra la existencia de ese "saber específico" que requeriría la profesión periodística. Sin embargo, un pensamiento compartido entre periodistas y empresarios de los medios es que la carrera por sí misma no garantiza nada, que las herramientas del oficio se aprenden en la práctica y se fortalecen con la misma. ${ }^{2}$

La interpretación de este sentir generalizado en el medio periodístico podría llevar a creer que las escuelas no han cumplido con su deber de formar a los periodistas "como debe ser". No obstante, el fenómeno es mucho más complejo, pues si bien es cierto que la desvinculación "entre lo que necesitan los empleadores y lo que ofrecen las universidades" 3 es un problema grave no sólo en comunicación sino en toda la educación superior en México, en el caso del periodismo las empresas informativas desempeñan un papel preponderante en la definición de las prácticas que predominan en este campo.

El propósito de este artículo es sencillamente reconstruir la trayectoria de la enseñanza del periodismo en México, como un antecedente

${ }^{1}$ Esta especie de demanda es manifestada por ciudadanos comunes, por políticos, por analistas, e incluso por los propios periodistas.

${ }^{2}$ Comunicación personal con el periodista Pedro Mellado (Guadalajara, 1995).

${ }^{3}$ Comunicación personal con Lázaro Ríos, director editorial del Grupo Reforma (ciudad de México, febrero 1996). 
necesario para discutir (en un trabajo posterior) el tema de la llamada "profesionalización" de este oficio.

No se trata de una crítica a las escuelas de comunicación como tales, cuyos proyectos y problemas son más amplios que el de la formación de periodistas. Lo que se quiere señalar mediante la descripción de los problemas reconocidos incluso por las propias escuelas, es la falta de una definición de la sociedad misma frente al periodismo, de las funciones que debería cumplir, y del perfil deseable en un periodista universitario. Tal indefinición está ligada, sin duda, al funcionamiento general del sistema económico, político y cultural de la sociedad mexicana.

\section{LA ENSEÑANZA DEL PERIODISMO EN MÉXICO}

Para ejercer el periodismo en México no se necesita una acreditación formal de que se es periodista, ni un título universitario específico del campo del periodismo o de la comunicación social.

Si bien es cierto que en la actualidad se tiene registro de al menos 240 programas de formación universitaria relacionados con el campo de la comunicación, ${ }^{4}$ y que cada vez son más los medios que prefieren contratar a egresados o estudiantes universitarios de estas carreras, no hay exigencias institucionales que impidan a personas con otro tipo de formación trabajar en las redacciones de los medios informativos; incluso, en muchas ocasiones los directivos de los medios prefieren contratar a gente que no haya estudiado comunicación o periodismo.

Durante las primeras décadas del periodismo comercial en México, los periodistas aprendieron el oficio ejerciéndolo, y algunos se legitimaron como periodistas "de referencia", bien por la influencia que adquirieron en sus roces con las esferas del poder, o por su estilo crítico y su vigilancia de los poderosos. De muy pocos periodistas se puede decir que "hicieron escuela".

La enseñanza formal del periodismo en México se inició antes de la Segunda Guerra Mundial, con una escuela patrocinada por la Universidad Femenina. Sin embargo, tal oferta no tuvo peso ni aceptación (Baldivia 1981: 19), por lo que se considera que la primera escuela reconocida de periodismo en México fue la Carlos Septién García, que exis-

\footnotetext{
${ }^{4}$ Según la base de datos de la Asociación Nacional de Universidades e Instituciones de Educación Superior. http://www.anuies.mx/perl/anuies/planes/ repplan.pl
} 
te hasta nuestros días, y que se fundó en la ciudad de México en 1948. ${ }^{5}$ Después de "la Carlos Septién", la Universidad Nacional Autónoma de México (UNAM) abrió en 1951 una licenciatura en periodismo adscrita a la Facultad de Ciencias Políticas y Sociales, y en 1954 nació la Escuela de Periodismo de la Universidad Veracruzana (Luna 1991: 11; Andión 1991: 56).

La cronología de las escuelas pioneras de periodismo en México es así de breve, pues a partir de 1960, con la fundación de la primera carrera de Ciencias y Técnicas de la Información en el país por la Universidad Iberoamericana, será difícil diferenciar las carreras específicamente orientadas a la formación de periodistas, de aquellas cuya intención es formar "comunicadores sociales", con un perfil "polivalente" (Marques de Melo 1988: 6; Andión 1991: 55). ${ }^{6}$ Si bien el Instituto de Filosofía Pío XII en Guadalajara, hoy Universidad del Valle de Atemajac (Univa), fundó en 1962 una escuela de periodismo a nivel técnico, la primera en el Occidente de México, más tarde cambiará su oferta por la de Ciencias y Técnicas de la Comunicación, y lo mismo ocurrirá con las carreras de periodismo de la UnAm y de la Universidad Veracruzana. Carlos Luna sostiene que con la carrera de Ciencias de la Comunicación de la UIA se "funda en el país un nuevo modo de entender y de enfrentar educativamente las exigencias y posibilidades comunicacionales de la sociedad" (1991: 11-12), un modelo cualitativamente distinto a los orientados hacia el periodismo.

Considerando que en Brasil y Argentina este tipo de escuelas surgieron desde los años treinta (Marques de Melo 1988; Andión 1991), podemos afirmar, entonces, que las escuelas de periodismo se iniciaron tardíamente en México y que antes de que se construyera un modelo, o de que se generara una tradición de enseñanza del periodismo en el país, esta función fue absorbida por las nacientes "escuelas de Ciencias y Técnicas de la Información" o de "Ciencias de la Comunicación", coincidiendo con la tendencia impuesta en América Latina por la UNESCO, por medio del Centro Internacional de Estudios Superiores de Periodismo para América Latina (CIESPAL).

\footnotetext{
${ }^{5}$ Algunos autores señalan el año de 1949 como el del nacimiento de la Escuela Carlos Septién García; la propia institución sitúa su fundación en 1948 (cf. www.septien.edu.mx).

${ }^{6}$ Según José Marques de Melo, el comunicador polivalente era "el profesional apto para desempeñar cualquier actividad dentro de la industria cultural" [...], un concepto asimilado "en forma distorsionada del modelo norteamericano de la School of Mass Communication".
} 


\section{El "Plan tipo" Del CIesPal}

A finales de la década de los cincuenta, la UNESCO encuentra un "descompás entre las estrategias de uso de los medios de comunicación y los programas de desarrollo de la región", por lo que, junto con la OEA y las fundaciones internacionales Ford y Friedrich Ebert, auspicia la creación del CIESPAl (Marques de Melo 1993: 38; Andión 1991: 55) .

Este organismo promovería en la región latinoamericana "un 'Plan tipo' de escuela de ciencias de la información colectiva, con la intención explícita -dice Mauricio Andión- de subsanar la brecha existente entre las escuelas tradicionales de periodismo y las necesidades teóricas y prácticas derivadas de la emergencia de los medios electrónicos de comunicación" (1991: 55). ${ }^{7}$

Marques de Melo afirma que, con su "Plan tipo" propuesto en 1964, el CIESPAL empujó a las escuelas latinoamericanas de periodismo y comunicación a un "callejón sin salida", con su concepción del profesional polivalente, que amalgamaba en su curricula diversos conocimientos técnicos para el manejo de los media con una concepción positivista de la teoría de la comunicación (Marques de Melo 1988: 6).

La equivocación del CIESPAL -dice en otro momento Marques de Melo- fue idealizar un modelo de formación apropiado para pequeñas comunidades, principalmente rurales, donde la sociedad requería un profesional habilitado para ejercer múltiples y diversas tareas comunicativas. Estimular su implantación en forma indiscriminada en todo el continente significó un retroceso. La universidad se distanció de la sociedad. Los profesionales no egresaban habilitados para el desempeño de funciones especializadas (1993: 38).

En opinión de Andión (1991), el modelo del CIEspal era conceptualmente ambiguo, y "provocó que cada escuela le otorgara un significado particular." Entre las múltiples recomendaciones propuestas por el CIESPAL, las más influyentes, de acuerdo con este autor, fueron:

1. Las escuelas deberían tener nivel universitario.

2. El programa académico debería durar un mínimo de cuatro años.

3. El programa de estudios debería incluir cursos humanísticos y técnico-profesionales.

4. Las escuelas deberían tratar de convertirse en facultades autónomas dentro de las universidades.

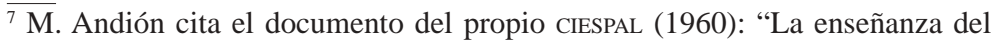
periodismo y los medios de información". 
5. Al extender sus ramos, las escuelas deberían convertirse en escuelas de ciencias de la información.

\section{LA INVESTIGACIÓN SOBRE LA FORMACIÓN DE PERIODISTAS EN MÉXICO}

Documentar el desarrollo, evolutivo o simplemente cronológico, de la formación de periodistas en México, es una tarea difícil, pues la rápida adopción en el país del modelo de "escuelas de comunicación" explica hasta cierto punto la escasez de trabajos académicos sobre la enseñanza del periodismo, ya que la mayor parte de la investigación o reflexiones al respecto abordan la problemática, mucho más amplia y ambigua, de la enseñanza de la "comunicación", y esto sucede aún en artículos con títulos referentes al periodismo. El único documento que ha hecho mayor hincapié en el tema de manera específica es el de José Baldivia, $L a$ formación de periodistas en América Latina: México, Chile, Costa Rica, escrito en 1981 y auspiciado por el Centro de Estudios Económicos y Sociales del Tercer Mundo (CEESTEM) y por la Federación Latinoamericana de Periodistas (Felap). El libro aparece en el contexto de las discusiones sobre el Nuevo Orden Informativo Internacional (NOII) y del debate sobre la reglamentación del Derecho a la Información, como parte de la reforma política impulsada por el presidente José López Portillo (cf. Luna 1991: 42).

Inevitablemente, el análisis de Baldivia se apoya también en el de las escuelas de comunicación existentes hasta entonces; la parte central relacionada con la enseñanza del periodismo, señala Carlos Luna, es el reporte de una encuesta aplicada a 21 instituciones educativas. Sin embargo, concluye este autor, el análisis de Baldivia sobre los planes de estudio y los antecedentes de las escuelas, traducido en "modelos" de carrera, poco informa sobre la calidad de la enseñanza de las mismas ( $c f$. Luna 1991: 45). ${ }^{8}$

El trabajo de José Baldivia fue muy criticado en el medio académico mexicano, por la ligereza de algunas afirmaciones, la clasificación de información sobre bases insuficientes, la falta de matices en las conclu-

${ }^{8}$ Como lo señala Carlos Luna, Baldivia genera una tipología de modelos de carrera a partir de los cuales clasifica las opciones existentes: modelo teórico-sociológico, con sus variantes cibernetista y cuestionador, y el modelo profesionalista, con sus variantes acrítico y crítico. 
siones, y por un cierto prejuicio en contra de las universidades privadas, sostiene Luna (1991: 46). No obstante, algunas de las afirmaciones de Baldivia en su estudio sobre la enseñanza del periodismo en México parecen válidas si se considera la mirada de otros investigadores, afirmaciones que expondré en el apartado "La problemática eterna de las escuelas de comunicación".

\section{Las escuelas de COMUniCación en MéXico}

Por lo anterior, hablar de la formación de los periodistas en México nos remite necesariamente a la discusión acerca de la formación de "comunicadores", y por ello es pertinente dar algunos datos sobre las escuelas de comunicación en el país, aunque sin entrar en las características de los curricula, ni analizar en forma exhaustiva la problemática más amplia de estas escuelas.

En su origen, la enseñanza de la comunicación en México estuvo centrada en el periodismo escrito. A partir de la apertura de la carrera de Ciencias de la Comunicación en la UIA (1960), que suponía un modelo académico más amplio que el de la prensa escrita, el desarrollo de la carrera en México toma esta tendencia. Incluso dos de las tres escuelas de periodismo prexistentes se sumarían más tarde a este modelo: la de la UNAM y la de la Universidad Veraruzana, ampliando su curricula para dar cabida a los medios electrónicos (Luna 1991: 32; Fernández 1991: 95).

Para los estudiosos del fenómeno, el surgimiento y desarrollo de las escuelas de comunicación en México y en América Latina se explica "como la respuesta natural del sector educativo a la introducción en el continente de las nuevas tecnologías de comunicación", para lo cual tuvieron que aceptar la intervención del CIESPAL, cuyo objetivo, como el mismo organismo lo subrayaba, era "llenar el vacío existente entre las escuelas de periodismo y el boom de los medios electrónicos" (Luna 1991: 46-47). ${ }^{9}$

En el mismo sentido, Fátima Fernández afirma que el surgimiento de las escuelas de comunicación en América Latina coincide con el advenimiento del invento de la televisión, durante la década de los cincuenta, y agrega que

${ }^{\overline{9}}$ La afirmación se deriva de la revisión que hace Luna de los trabajos: La ciencia de la comunicación en América Latina, de Heliodoro Jiménez, y La ciencia de la comunicación en México. Desarrollo y situación actual, de Manuel Corral C. y Heliodoro Jiménez. 
La televisión ha sido el medio de difusión que más preocupación ha despertado entre los educadores y el que más ha influido en la creación de escuelas de comunicación, al menos esa lectura puede hacerse de los tiempos en que comienzan a proliferar las pantallas en las casas y los estudios de comunicación en las universidades (Fernández 1997: 94).

\section{EL CRECIMIENTO DESMESURADO EN EL NÚMERO DE ESCUELAS DE COMUNICACIÓN}

Al empezar la década de los cincuenta, había en América Latina 13 escuelas dedicadas a la formación de especialistas para los medios de comunicación (Marques de Melo 1988); de ellas sólo la Escuela de Periodismo Carlos Septién García se ubicaba en México. Hacia 1960 eran alrededor de 35 escuelas en la región latinoamericana (Prieto 1988), sólo tres situadas en México: la Carlos Septién, la de la unam y la de la U. Veracruzana. Sin embargo, al paso de cada década, el número de planteles se duplicaba, de manera que hacia 1988 Felafacs registraba ya 190 establecimientos en Latinoamérica, con Brasil y México a la cabeza como países con mayor número de escuelas de comunicación: México tenía 42 y Brasil 71, según reporta José Marques de Melo (1988: 5-6). Un año después, Mauricio Andión encontraría en documentos de la misma Federación Latinoamericana de Facultades de Comunicación Social, que hasta 1989 existían en América Latina un total de 226 instituciones (1991: 56). Carlos Luna dice, por su parte, que en 1980 México contaba con 34 escuelas de comunicación, y en 1988 ya existían por lo menos 82 (1991: 10-11). Hacia 1999 ya se tenía registro en México de al menos 218 escuelas de comunicación (Prieto 1988; Benassini 2001). Finalmente, para constatar el crecimiento desmesurado del número de esta oferta educativa en el país, Raúl Fuentes señalaba hace poco más de una década (1992) que un tercio de las aproximadamente 244 escuelas de periodismo y comunicación que registraba la Felafacs en América Latina hacia 1990, se ubicaban en México (82 escuelas), y al día de hoy (2004) la base de datos de ANUIES (Asociación Nacional de Universidades e Instituciones de Educación Superior) tiene registro de 240 instituciones en México que imparten alguna modalidad de las licenciaturas en comunicación. ${ }^{10}$

$\overline{{ }^{10}}$ Dato citado por Caridad Hernández en la publicación electrónica Sala de Pren$s a$, núm. 63, y confirmado en búsqueda personal. $C f$. http:// www.saladeprensa.org y http://www.anuies.mx 
CUADRO 1

NúMERO DE ESCUELAS DE COMUNICACión EN AMÉRICA LATina Y MÉXICO

\begin{tabular}{|c|c|c|c|}
\hline Año del registro & $A L$ & México & Autor consultado* \\
\hline 1950 & 13 & - & Marques de Melo, 1988 \\
\hline 1950 & - & 1 & Escuela Carlos Septién \\
\hline 1960 & 44 & - & Raymond Nixon, 1982 \\
\hline 1960 & 35 & 3 & Daniel Prieto, 1988 \\
\hline 1964 & - & 4 & Raúl Fuentes, 1988 \\
\hline 1970 & 81 & - & Raymond Nixon, 1982 \\
\hline 1974 & - & 13 & Raúl Fuentes, 1988 \\
\hline 1975 & - & 21 & Mauricio Andión, 1991 \\
\hline 1980 & 163 & - & Raymond Nixon, 1982 \\
\hline 1980 & - & 34 & Carlos Luna, 1991 \\
\hline 1982 & - & 36 & Carlos Luna, 1995 \\
\hline 1984 & - & 47 & Raúl Fuentes, 1988 \\
\hline 1985 & - & 60 & Carlos Luna, 1991 \\
\hline 1988 & - & 82 & Carlos Luna, 1991 \\
\hline 1989 & 226 & - & Mauricio Andión, 1991 \\
\hline 1990 & 244 & 82 & Raúl Fuentes, 1992 \\
\hline 1999 & - & 218 & Claudia Benassini, 2001 \\
\hline 2004 & - & 240 & Caridad García y ANUIES \\
\hline
\end{tabular}

${ }^{*}$ Las obras de los autores mencionados aparecen en la bibliografía. En pocos casos los autores dan cifras tanto de México como de América Latina, razón por la que aparece el mismo año de registro en más de una ocasión.

Fuente: propia.

No sorprenden las diferencias entre los datos de unos y otros investigadores ya que, como todos ellos lo han manifestado, se trata de datos siempre aproximativos porque ni antes ni ahora se ha tenido registro de todas las escuelas y academias que podrían haber estado operando en el país.

En su primera etapa, las escuelas mexicanas de comunicadores estaban fundamentalmente en manos de instituciones de educación superior de carácter privado. Según Carlos Luna, en 1974 se inició la participación del sector público en este campo de enseñanza, que fue un año "especialmente prolífico" en la apertura de escuelas de comunicación (1991: 13). Mauricio Andión documenta, precisamente, que en 1974 se establecen las licenciaturas en comunicación de: la Universidad Autónoma de Nuevo León (UANL), la Universidad Autónoma de Sinaloa (UAS), la Universidad Autónoma Metropolitana-Xochimilco (UAM-X) y las de las escuelas profesionales de Acatlán y Aragón (ENEP). 


\begin{tabular}{|c|c|c|c|c|}
\hline \multicolumn{5}{|c|}{ CuAdro 2} \\
\hline Periodo & Núm. de carreras & $\%$ del total & $A \tilde{n} o$ & Número \\
\hline $1949-1968$ & 7 & 3.27 & & acumulativo \\
\hline $1969-1974$ & 12 & 5.50 & & \\
\hline $1975-1979$ & 13 & 5.99 & 1968 & 7 \\
\hline $1980-1984$ & 30 & 13.82 & 1974 & 19 \\
\hline $1985-1989$ & 30 & 13.82 & 1979 & 32 \\
\hline $1990-1994$ & 63 & 28.80 & 1984 & 62 \\
\hline $1995-1999$ & 63 & 28.80 & 1989 & 92 \\
\hline 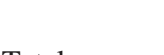 & & & 1994 & 155 \\
\hline Total & 218 & 100.00 & 1999 & 218 \\
\hline
\end{tabular}

Fuente: Claudia Benassini, Diálogos de la Comunicación, núm. 62, Felafacs, julio 2001, p. 45.

Además, apunta Andión, alrededor de esos años la UNAM comienza el proceso de revisión que culmina en la reorientación de la carrera de periodismo hacia el modelo de ciencias y técnicas de la información, "más acorde con las directrices ciespalinas" (1991: 56).

A pesar de la variación en las cifras proporcionadas por los diferentes autores, la década de los ochenta es evidentemente el primer periodo que registra el incremento más significativo en el número de escuelas en México: Carlos Luna reportaba a principios de los noventa que entre 1980 y 1988 se crearon al menos 48 nuevas ofertas para la enseñanza de la comunicación, la mayoría en universidades de carácter privado, algunas de las cuales abrieron la carrera en diferentes estados de la república, como extensiones de la universidad matriz: son los casos del ITESM, la UIA, la Universidad del Valle del Bravo, la Universidad del Valle de México y la Universidad de Occidente (Luna 1991: 14-15). Según el dato más reciente al respecto (Benassini 2001), el número de escuelas que surgieron en la década de los ochenta fue de 60 (ver Cuadro 2).

A este fenómeno expansivo, prácticamente sin control, se le conocería en México y América Latina como el "boom de las escuelas de comunicación", fenómeno que se agravaría en los últimos 15 años, pues entre 1990 y 2003 el número de escuelas de comunicación en México de las que se tiene registro aumentaría de 82 a 240, esto es, casi en $300 \%$.

Claudia Benassini presenta un cuadro sobre el crecimiento en el número de carreras de comunicación por quinquenios a partir de 1969, y señala de manera especial un periodo inicial que va de 1949 a 1968. A 


\section{CuAdRo 3}

Porcentajes de escuelas de Comunicación o Periodismo PÚBLICAS Y PRIVADAS EN MÉXICO Y POBLACIÓN ESTUDIANTIL EN DOS PERIODOS 1988

$\%$ tipo de escuela Población estudiantil\% Fuente documental

$\begin{array}{lll}\text { 26.0 Públicas } & 57.5 & \text { Luna (1991) } \\ \text { 74.0 Privadas } & 42.5 & \end{array}$

1999

$\begin{array}{lll}15.8 \text { Públicas } & 46.9 & \text { Benassini (2001) }\end{array}$

84.2 Privadas $\quad 53.1$

Fuente: Luna 1991; Benassini 2001.

dicho cuadro le agrego un elemento (número acumulativo) que permite visualizar el número total de escuelas en cada fin de periodo (ver Cuadro 2).

En cuanto al carácter público o privado de las ofertas educativas, datos de 1988 muestran que 74\% de las escuelas eran privadas y $26 \%$ públicas, aunque la distribución de la población estudiantil era en sentido inverso: $57.5 \%$ en universidades públicas y $42.5 \%$ en universidades privadas (Luna 1991: 23).

Las cifras más actuales al respecto corresponden al estudio de Claudia Benassini (2001), quien afirma que de las 240 escuelas de comunicación o periodismo identificables hasta entonces, únicamente 38 pertenecían a universidades públicas, y las 202 restantes eran de carácter privado. Las escuelas públicas correspondían a $15.83 \%$ del total y atendían a $46.9 \%$ de la población estudiantil, mientras que en las escuelas privadas, $84.16 \%$ del total albergaban a $53.1 \%$ de los alumnos de comunicación o periodismo (ver Benassini 2001, Cuadro 3). ${ }^{11}$

\section{LA POBLACIÓN ESTUDIANTIL Y LOS EGRESADOS}

El aumento en el número de ofertas educativas en comunicación se traduce, obviamente, en cifras crecientes de alumnos y de egresados. Tan

${ }^{11}$ Sobre el mismo tema, Caridad Hernández presenta datos de 2003 con cifras no coincidentes: de acuerdo con esta autora, de las 240 licenciaturas registradas actualmente en la base de datos de ANUIES, $24 \%$ son de carácter público y 178 de carácter privado (74.16\%). Cf. Sala de Prensa 63, enero 2004, http://www.saladeprensa.org 
sólo en el periodo de 1984 a 1988 la matrícula en las escuelas de comunicación en México pasaría de 14 mil a 25 mil estudiantes (Fuentes 1988: 38; Andión 1991). Carlos Luna reporta para 1988 al menos 27 mil alumnos en 82 escuelas y aproximadamente 25 mil egresados de las 60 escuelas registradas hasta antes de 1985 (1991: 17). De acuerdo con Raúl Fuentes, hacia 1990 la población de las escuelas de periodismo y comunicación que se ubicaban en México en ese año era de alrededor de 34 mil estudiantes (un tercio de los 100 mil reportados para AL), y los comunicadores universitarios egresados a la misma fecha serían una cantidad semejante. Las cifras siempre aproximativas (Fuentes 1992: 17).

Hacia 1995, los estudiosos del fenómeno se manifestaban preocupados porque la expectativa era que "en la segunda mitad de la década de los noventa, egresarían de las escuelas tantos comunicadores como en los pasados treinta años" (Luna 1991: 134); esto significaría sin duda más de 35 mil egresados de escuelas de comunicación en media década y para el 2000 un total aproximado superior a 70 mil comunicadores en todas sus versiones, desde la apertura de las primeras escuelas. ${ }^{12}$ Lo anterior sin contar "el dato impreciso de cerca de 50 mil alumnos inscritos" hacia finales del 2000, como lo reporta Claudia Benassini, quien hace ver que, de acuerdo con los datos del Anuario 2000 de la ANUIES, los estudios en comunicación se ubican entre los más solicitados a nivel nacional, ocupando en ese momento el octavo lugar (Benassini 2001). ${ }^{13}$

\section{¿Por qué tantas escuelas de comunicación?}

Se puede sostener que tanto la ambigüedad de los perfiles profesionales de las escuelas de comunicación, como una cierta "moda", han sido factores determinantes de la alta demanda de esta oferta educativa. En sus primeros años las escuelas superiores de periodismo nunca tuvieron tanta demanda como las de comunicación; Baldivia afirma que durante los dos primeros años de funcionamiento de la Escuela de Periodismo de la Universidad Nacional Autónoma de México, y como una promoción de

$\overline{12}$ Dato que deduzco de las cifras precedentes presentadas por Raúl Fuentes y Carlos Luna.

${ }^{13}$ Benassini señala que "Las estadísticas corresponden al Anuario 2000 de la Asociación Nacional de Universidades e Instituciones de Educación Superior (ANUIES), con las imprecisiones ya señaladas. El octavo lugar es después de Derecho, Contaduría, Administración, Ingeniería industrial, Medicina, Informática y Arquitectura, en ese orden" (2001: 45). 
la nueva oferta educativa, se dispensó a los periodistas con cinco años de ejercicio profesional de presentar título de bachiller para poder ingresar a dicha escuela (Baldivia 1981: 70). Además, durante los primeros años de vida de la carrera de periodismo en la UnAM, y bajo el modelo tradicional de enseñanza del oficio, el índice de deserción era hasta de $73 \%$, muy superior al de otras carreras, y muy escaso el número de estudiantes que se graduaban. Diez años después, cuando la unAm reorienta la enseñanza hacia un tratamiento más sociológico de los problemas de la comunicación, y con el boom comercial y publicitario que se producía en México, la población estudiantil se multiplicaría por ocho (Baldivia 1981: 70-72).

Para Carlos Luna, un estudio profundo de las razones de apertura de cada una de las escuelas podría llevar a explicaciones como las siguientes (1991: 18-19):

- Rivalidades interinstitucionales.

- Competencia a nivel local.

- Respuesta a demandas específicas de grupos empresariales (vinculados a ciertas universidades).

- Promoción de ideologías mediante formación de recursos humanos.

- Apertura de espacios para juego político en las universidades.

- Atención a problemas de comunicación regionales.

Aunque también se puede considerar como explicación al boom de las escuelas, el factor de la expansión de los medios en un cierto periodo: es el caso de la prensa en México, que creció de 197 a 348 periódicos en el periodo 1960-1985, de los cuales $40 \%$ se concentraba en la ciudad de México (Andión 1991: 57).

\section{LAS ORIENTACIONES PREDOMINANTES}

De acuerdo con Fuentes, la formación universitaria en el campo de la comunicación a principios de los noventa, estaba constituido por "elementos superpuestos, casi nunca consistentemente integrados pero simultáneamente vigentes, de tres modelos fundacionales de la carrera, que remiten a tres proyectos diferentes": el de la formación de periodistas, el de comunicador como intelectual (con el que nace la primera escuela de Ciencias de la Comunicación en México, en la U. Iberoamericana), y el de comunicólogo como científico social. 
El más antiguo de los modelos, el de la formación de periodistas, es también el más fuertemente arraigado en nuestras escuelas, aun en aquellas que fueron fundadas ya como escuelas de comunicación. Puede decirse que, más de cincuenta años después del mítico origen latinoamericano en La Plata, en la mayor parte de las instituciones, el objeto de estudio y su abordaje tanto en la enseñanza como en la investigación universitarias, están primariamente compuestos por representaciones — quizá cada vez más refinadas y por ello cada vez más exclusivas - de las prácticas periodísticas (Fuentes 1992: 21-22).

El llamado modelo de la formación de periodistas, apunta Fuentes, estuvo "inspirado originariamente por Pullitzer e impulsado por el CIESPAL de los sesenta". El modelo del comunicador como intelectual se distinguía del anterior por su acento en la solidez de la formación intelectual basada en las humanidades, "ante la cual la habilitación técnica estaría subordinada, pero de tal manera que garantizara la capacidad para acceder, a través de los medios, a la transformación de la dinámica sociocultural conforme a marcos axiológicos bien definidos" (ibidem).

Carlos Luna da cuenta de que hacia 1988 la carrera de comunicación se ofrecía bajo 25 nombres por lo menos, y de que de las 82 escuelas registradas en su estudio, sólo cuatro expedían un título profesional de "Licenciado en Periodismo", aunque en otras escuelas de cc la orientación predominante favoreciera también la formación de periodistas (1991: 33). Ciertamente, una de las tres especialidades profesionales predominantes en las carreras que declaran alguna opción terminal, es la de periodismo; las otras dos son publicidad y relaciones públicas (Luna 1991: 34; Benassini 2001: 44).

Según el análisis de Caridad García en 2004, y con la información de los sitios disponibles de escuelas de comunicación (ella accedió a 64 páginas de las universidades que tienen la carrera de comunicación), se pueden encontrar hasta 20 nombres diferentes bajo los cuales se denomina a la carrera, a saber:

1. Comunicación.

2. Ciencias de la comunicación.

3. Comunicación audiovisual.

4. Comunicación corporativa.

5. Comunicación social.

6. Comunicación y publicidad.

7. Comunicación y relaciones públicas. 
8. Comunicación y tecnologías educativas.

9. Periodismo.

10. Publicidad y comunicación.

11. Comunicación organizacional.

12. Medios masivos de comunicación.

13. Comunicación e información.

14. Ciencias de la información y de la comunicación.

15. Comunicación académica.

16. Periodismo y comunicación.

17. Comunicación visual.

18. Comunicación multimedia.

19. Comunicación humana.

20. Ciencias y técnicas de la comunicación.” (Véase García 2004) ${ }^{14}$

En la actualidad la Asociación Nacional de Universidades e Institutos de Educación Superior (ANUIES) da cuenta de la existencia de 24 carreras o programas de estudio en México que incluyen la formación de periodistas de manera explícita, ${ }^{15}$ aunque sólo ocho de ellas son exclusivamente de periodismo, y las restantes se suman a la larga lista de escuelas de comunicación, alguna de cuyas orientaciones formativas es el periodismo.

\section{DISTRIBUCIÓN GEOGRÁFICA}

Hacia 1988, las 82 carreras de comunicación de las que se tenía registro se localizaban en 40 ciudades de 23 estados de la república, aunque distribuidas de manera muy desequilibrada: tan sólo en la ciudad de México se situaba más de $25 \%$ del total de establecimientos (21 escuelas), con la particularidad de que en esta entidad se concentraba casi $50 \%$ de la población estudiantil. Tan sólo la UNAM albergaba a cerca de $30 \%$ de estudiantes de comunicación al nivel nacional; mientras que en el sur y sureste del país (Guerrero, Oaxaca, Chiapas, Tabasco, Campeche, Yucatán y Quintana Roo), había sólo seis carreras de comunicación, con una población estudiantil aproximada de $2.6 \%$ del total nacional (Luna 1991: 20). Para descentralizar y redistribuir a la población estudiantil

${ }^{14}$ Claudia Benassini, por su parte, sostiene que son más de 30 los nombres de la carrera en la actualidad (2001: 45).

${ }^{15}$ Dato obtenido mediante el buscador de la dirección electrónica de la ANUIES. http://www.anuies.mx/perl/planes/repplan.pl 
interesada en la carrera de periodismo, en 1975 la UNAM abrió programas de periodismo y comunicación colectiva en las Escuelas de Estudios Profesionales (ENEP) Acatlán y Aragón, con el mismo plan de estudios de la Facultad de Ciencias Políticas y Sociales. El proceso de expansión de las escuelas de comunicación se incrementó en los años ochenta en las universidades privadas, por fines distintos a los de la unam: el Instituto Tecnológico de Estudios Superiores de Monterrey es el caso más sobresaliente, pues inició en 1971 y a la fecha ofrece la carrera en 11 ciudades, bajo la estructura del sistema ITESM. Por su parte, la Universidad Iberoamericana tiene cinco sedes y trabaja en conjunto con el ITESo en el llamado sistema UIA-ITESo (Luna 1991). ${ }^{16}$ Hoy en día se puede dar cuenta de un número mayor de universidades privadas que extendieron sus servicios por medio de redes a nivel nacional, pero las del ITESM y de la UIA son las que tienen mayor tradición y reconocimiento en el mercado profesional.

\section{LA PROBLEMÁTICA ETERNA DE LAS ESCUELAS DE COMUNICACIÓN}

La creación de las Facultades de Comunicación muestra simplemente la objetivación de ciertos saberes y conocimientos que constituían las prácticas de comunicación que ya existían y su traducción a un plan de estudios específico.

Raúl Fuentes (1991: 39).

Consultando la fuente documental más rica en México en materia de investigación sobre la enseñanza de la comunicación, el Centro de Documentación CONEICC, Carlos Luna realizó una revisión de los documentos disponibles a finales de los ochenta que dieran cuenta de la reflexión, análisis y propuestas más importantes acerca de la formación profesional de comunicadores sociales en México (Luna 1991: 39). De acuerdo con Luna, el diagnóstico de mayor rigor y fundamentación sobre la enseñanza de la comunicación en México, fue uno emprendido por la Coordinación General de Comunicación Social de la Presidencia de la República, durante el gobierno de José López Portillo (1976-1982). En ese diagnóstico participaron alrededor de 70 personas, con resultados de unas 6 mil 500 páginas con información y análisis de gran valor. El

$\overline{{ }^{16}}$ Originalmente son datos de Carlos Luna publicados en 1991, pero los he actualizado o corroborado a través de los sitios de Internet de las universidades citadas. 
trabajo se realizaba en el marco del debate nacional en torno a la reglamentación del Derecho a la información, a principios de los ochenta (op. cit.: 49-50). El debate nunca se publicó. Parte de la información generada por el diagnóstico referido fue rescatada por algunos académicos miembros del equipo que lo elaboró, entre ellos Beatriz Solís quien, apoyándose en el análisis de los contenidos de los planes de estudio y de la operación curricular general, criticó la continua revisión de los planes de estudio de las carreras de comunicación en México, "sin base objetiva y subordinada a los factores administrativos", y la fragmentación de la enseñanza que genera la formación de profesionales "con un conocimiento parcelado de la realidad y que ubica su práctica en un esquema igualmente parcializado". ${ }^{17}$

\section{Sin modelo y sin perfil}

Sin duda el cuestionamiento más frecuente a las escuelas de comunicación en México, a lo largo de su historia, ha sido el de su falta de definición académica, esto es, el de la ambigüedad de sus perfiles ( $c f$. Solís 1983; Prieto 1988; Fuentes 1988; Luna 1991; Caletti 1991; Godoy y Herrán 1996), un problema que prevalece a la par del de intentar adaptarse a las necesidades del mercado laboral tal y como es, ${ }^{18}$ lo que se traduce siempre en un desfase entre la enseñanza y la práctica, debido sobre todo a la inacabable carrera contra las novedades tecnológicas en el campo de las comunicaciones.

Hace más de 20 años Baldivia señalaba, entre sus críticas a la enseñanza de la comunicación en México, que

La inmensa mayoría de las instituciones que brindan la carrera tiene como objetivo abastecer de cuadros profesionales a los medios tal como ellos son (1981: 76; $c f$. Luna 1991: 45).

${ }^{17}$ Por decisión presidencial, reporta Luna, el debate se suspendió y se resolvió por la no-reglamentación. El coordinador de Comunicación de la Presidencia renunció y el debate nunca fue publicado. $C f$. Luna, op. cit. p. 54, en donde cita a Beatriz Solís 1983, p. 59.

${ }^{18}$ Es importante recordar que alrededor de $84 \%$ de las escuelas son privadas, aunque también las universidades públicas sucumben ante las presiones del mercado. 
Raúl Fuentes refrendaría más tarde esta preocupación, agregando que la influencia del mercado en las escuelas para la definición del perfil del comunicador llevaba a un

[...] desplazamiento creciente de la carrera con respecto a la dinámica social y una notable pérdida del objeto académico en lo científico y en lo profesional. Y esto aunado a un explosivo crecimiento del número de instituciones y de estudiantes de comunicación en los últimos años, [confundía] enormemente el campo, acercando la situación a un verdadero caos, sin sentido y sin remedio (1988: 37 ).

Hacia 1996, y a partir de las conclusiones y recomendaciones que hacía la Federación Latinoamericana de Facultades de Comunicación Social (Felafacs) a las escuelas de la región, Godoy y Herrán sintetizarían ciertas constataciones en el desarrollo histórico de las escuelas de comunicación de América Latina, que son totalmente válidas para el caso de México:

Las escuelas pioneras de periodismo trataron de responder a las demandas del mercado desde un concepto restringido de la comunicación.

Con la transformación de las escuelas de periodismo en facultades de comunicación se da una cierta apertura hacia el ámbito de las ciencias sociales [...] “Apertura que multiplica o diversifica el perfil del comunicador, al precio de una inseguridad que desembocó —en muchos casos- en una miope percepción de las demandas sociales."

"En la actualidad podemos observar una apertura desmedida cuya consecuencia ha sido la falta de definición académica de las escuelas." (1996: 16).

Las opiniones de los investigadores en diferentes momentos, confirman el problema de la amplitud de los perfiles, o de la ambigüedad de las ofertas educativas de las licenciaturas en comunicación de América Latina. En los ochenta, Mauricio Antezana, citado por Fuentes (1991) sostenía que ante la indefinición, en las escuelas de comunicación

[...] el objeto académico se dilató y de un contenido eminentemente periodístico-informativo, se convirtió en un espectro en el que se podía incluir desde técnicas operativas puras hasta reflexiones teoréticas heterogéneas. ${ }^{19}$

${ }^{19}$ Fuentes, Raúl (1991: 38), en donde cita el trabajo de Mauricio Antezana: "La errátil circunstancia de la ciencias de la comunicación". Ponencia en la II Reunión Nacional de Investigadores de la Comunicación, AMIC, México, 1982, p .5. 
A principios de los noventa, Sergio Caletti concluía que:

Nuestras carreras tienden a tratar de abarcar, resolver y dar cuenta de más problemas y realidades de las que cualquier carrera razonablemente puede. Desde la estética hasta la economía, desde el sonidista hasta el periodista deportivo o el gerente de comunicaciones, desde el analista crítico hasta el productor publicitario o el comunicador alternativo (1991: 36).

En el mismo periodo, Raúl Fuentes señalaba que la mayoría de las escuelas latinoamericanas no contaban con información suficiente sobre las condiciones en que sus egresados se incorporaban al ejercicio profesional, ni sobre las tendencias decadentes, predominantes o emergentes, como información pertinente para orientar sus diseños curriculares... y lo más grave, no se conocían iniciativas para resolver esa carencia:

Más bien, parece volver a tomar fuerza la tendencia a declarar inexistente el problema, y en consecuencia adoptar las maneras más eficientes de subordinar la formación universitaria a las demandas, explícitas y tácitas, de los empleadores, es decir, de quienes controlan el "mercado", casi siempre los mismos que controlan los medios de difusión masiva. De manera que aunque la reducción de "profesión" a "mercado de trabajo" y de "formación universitaria" a "adiestramiento funcional" es vista ahora como más "natural" y "práctica", no por ello la consideramos menos inaceptable (1991: 38).

Desde su fundación en junio de 1976, y a lo largo de sus 27 años de vida, el Consejo Nacional para la Enseñanza e Investigación de las Ciencias de la Comunicación (CONEICC), ha intentado discutir y analizar la problemática de las escuelas de comunicación del país, e intervenir mediante la reflexión académica en la planificación y orientación de las ofertas educativas. Sin duda la contribución del CONEICC ha sido valiosa para mantener la conciencia sobre la realidad de la enseñanza de la comunicación en México; sin embargo, sus recomendaciones no son de carácter normativo, y no todas las instituciones que abren escuelas pertenecen al organismo. Hoy se puede afirmar que la formación de los profesionales en comunicación presenta tantos problemas como concluían Beatriz Solís y otros académicos hace más de 20 años, y habría que agregar los retos y desafíos derivados de la irrupción de las nuevas tecnologías de la información y la comunicación en nuestra realidad social, y sus repercusiones en la enseñanza de las profesiones relacionadas con la comunicación. 


\section{La calidad de la enseñanza y falta de certificación}

En 1982 el número de escuelas en México era de 36, y en América Latina de poco más de cien. La Felafacs señalaba entonces la inminencia de graves problemas en el campo de la enseñanza de la comunicación si el crecimiento del número de escuelas continuaba al ritmo hasta entonces registrado: sobresaturación, falta de definición académica, deficiente calidad de la enseñanza (ver Luna 1991: 11). ${ }^{20}$

La señal de alerta se había emitido, pero el ritmo de crecimiento de las escuelas no sólo se mantuvo, sino que se multiplicó en proporciones escandalosas. En el año 2004 tan sólo en México se tiene registro de al menos 240 ofertas educativas en comunicación, y en América Latina de más de $600 .{ }^{21}$ Los problemas anunciados hace dos décadas son vigentes para el caso de México, y al parecer sin vistas de solución. Se siguen abriendo escuelas, sin estudios de mercados laborales, de pertinencia educativa, de criterios mínimos de calidad.

Todos los autores coinciden, desde el origen de los estudios sobre las escuelas de comunicación en México, en la falta de claridad de los criterios que orientan las decisiones para abrir una licenciatura en comunicación en México. Baldivia afirmaba en 1981:

No existe una planificación nacional sobre la base de un estudio de las necesidades del mercado de trabajo que evite la proliferación de escuelas de comunicación [...] se puede decir que el mercado se está saturando (p. 82).

${ }^{20}$ Carlos Luna cita el reporte de Felafacs La formación universitaria de comunicadores sociales en América Latina (1991: 9): "En la década de los años 80 , de mantenerse el ritmo de crecimiento, puede esperarse una crisis de gran magnitud por la sobresaturación de los mercados de trabajo, las dificultades de definición académica y la consecuente disminución en la calidad de la enseñanza. Estos problemas son ya notables en Brasil y México, países en que se concentra más del $60 \%$ de las escuelas de comunicación latinoamericanas".

${ }^{21}$ El sitio Infoamerica presenta un dato sin fecha de alrededor de 460 escuelas en América Latina. Sin embargo, Raúl Fuentes, en conversación personal (febrero 2004), sostiene que se conocen cifras, también aproximativas, de un mínimo de 200 escuelas de comunicación en Brasil, otras tantas en México, alrededor de 60 en Argentina, que sumándolas a las del resto de países de América Latina, nos permiten suponer la existencia de al menos 600 escuelas de comunicación en esta región del mundo. 
Diez años más tarde, Carlos Luna sostenía que

[...] la formación universitaria de comunicadores sociales no está territorialmente estructurada en función de demandas y necesidades locales y regionales, ni parece obedecer directamente a la constitución de los mercados de trabajo, sino que se encuentra atravesada por variables que escapan a una planificación en términos de respuesta a requerimientos sociales objetivos (1991: 20).

\section{A finales del 2000, Claudia Benassini coincidía y agregaba:}

Mucho menos obedece a políticas educativas que garanticen una planta docente debidamente capacitada y la mínima infraestructura adecuada para asegurar la formación de futuros profesionales en el campo (2001: 45-46).

Si bien las escuelas deben cubrir ciertos requisitos administrativos ante la Secretaría de Educación Pública para poder operar (remarquemos que alrededor del $84 \%$ de ellas son de carácter privado, según Claudia Benassini), no hay una instancia oficial que acredite la pertinencia y la calidad de planes de estudio para la formación tanto de comunicadores en general, como de periodistas en particular. Los criterios oficiales se pueden satisfacer en papel, pero la evaluación de la calidad en la enseñanza superior es algo muy reciente en el sistema educativo mexicano y además, se aplica principalmente a las instituciones públicas que requieren fondos federales para su funcionamiento. Adicionalmente, ya en la operación cotidiana de las escuelas, es aún vigente la conclusión a la que Baldivia llegó hace más de 20 años:

El bajo nivel de enseñanza, no sólo en periodismo y comunicación, sino en la educación superior en general, se debe a los bajos sueldos pagados a los profesores en las universidades públicas. ${ }^{22}$

Lo anterior explica, entre otras razones, el que la mayor parte de los profesionales de los medios que participan en la enseñanza del periodismo, lo hagan en las universidades privadas, en donde se les remunera de manera más justa e incluso se adaptan los cursos a su disposi-

${ }^{22}$ Como un ejemplo bastante ilustrativo, puedo mencionar el caso de una institución pública, la Universidad de Guadalajara, en la que a los profesores que sólo imparten una asignatura o curso a nivel licenciatura con carga de tres horas semanales, se les paga el equivalente a 36 dólares por mes. 
ción de tiempo, por ejemplo concentrando las horas de clase requeridas en seminarios intensivos.

Ciertamente, uno de los reproches más fuertes que hacen los periodistas a las escuelas de comunicación, es que muy pocos de los maestros que enseñan periodismo son o han sido periodistas, y algunos ni siquiera conocen una sala de redacción.

\section{La acreditación de las escuelas de comunicación}

La acreditación o certificación de la calidad de las escuelas superiores en México es todavía incipiente, y en el caso de las escuelas de comunicación o periodismo es aún un proyecto.

Existen documentos oficiales que hablan de acciones de planificación o evaluación desde finales de los años setenta, cuando el crecimiento de la educación superior en México fue explosivo. Sin embargo, todavía en la década de los noventa, las instancias federales continuaban buscando mecanismos para la evaluación de las universidades públicas y privadas, y generaron una figura para hacer evaluaciones diagnósticas y emitir recomendaciones (los CIEES), ${ }^{23}$ dejando al albedrío o interés de las propias universidades el "acreditarse", pues para ello, las instituciones deberían solicitarlo y cubrir el costo. La estrategia de la Secretaría de Educación Pública ha sido fomentar la constitución de organismos sociales que acrediten las ofertas educativas, vigilando, por medio de un organismo regulador que los procesos de acreditación apliquen "la norma nacional. ${ }^{24}$

En marzo de 2002, durante la LIII Asamblea del Consejo Nacional para la Enseñanza e Investigación de las Ciencias de la Comunicación, se integró una comisión de socios que tendrían la responsabilidad de estructurar un proyecto de Instancia de acreditación. Este proyecto fue presentado en octubre de 2003 durante la LVI Asamblea del Consejo, en medio de un ambiente de polarización de opiniones entre los miembros, que sugiere la posibilidad de que se postergue la inscripción del organismo acreditador ante las instancias federales.

${ }^{23}$ Comités Interinstitucionales para la Evaluación de la Educación Superior.

${ }^{24}$ En noviembre de 2000 se creó el Consejo para la Acreditación de la Educación Superior (COPAES) con el fin de regular los procesos de acreditación. $C f$. Acta de la LIII Asamblea del CONEICC. 


\section{EL LUGAR DE LAS ESCUELAS DE PERIODISMO Y COMUNICACIÓN EN LA FORMACIÓN DE PERIODISTAS EN MÉXICO}

Retomando las palabras que Daniel Prieto sostenía en 1988, también ahora se puede afirmar que el problema de la formación de periodistas en México y en América Latina aún no encuentra la respuesta más adecuada. "La polémica entre la capacitación a través de la práctica y la capacitación mediante el paso por las escuelas sigue vigente" (1988: 31). Las escuelas no dan los elementos que la práctica otorga, pero "la sola capacitación práctica no puede abarcar todas las posibilidades actuales de la comunicación, todas las demandas sociales" (p. 33). Curiosamente, Prieto hablaba en aquel tiempo de una "desprofesionalización" debida al "teoricismo, la falta de oficio y la poca especificidad de nuestros estudios" (p. 35). Hoy nos preguntamos simplemente si las escuelas han contribuido a la "profesionalización", en el sentido de un mejoramiento en la calidad de las prácticas periodísticas predominantes.

Entre periodistas experimentados, directivos y empresarios de los medios, es frecuente la opinión de que las escuelas de comunicación no dan al estudiante los elementos suficientes para ejercer el oficio periodístico. Consideran que la de comunicación es una carrera saturada, una profesión de moda, que la Secretaría de Educación Pública debería regular la apertura de escuelas que toman como negocio la educación de los estudiantes, que existe un exceso de reporteros sin especialización, que los egresados están mal preparados, que padecen de un gran rezago cultural, que a los estudiantes les falta ser más analíticos. Algunos afirman, incluso, que no creen en las escuelas de periodismo como lugar de formación, sino en la calle, en el periodista que aprende en la calle, pues es en el medio periodístico en donde muchos terminan aprendiendo hasta lo elemental de las técnicas de información. Señalan también que una de las razones de estas deficiencias en la enseñanza se deriva de la falta de vinculación entre medios y universidades, en donde la responsabilidad es de ambas partes (Salazar 1993: 60-64). ${ }^{25}$

La crítica desde el punto de vista de los académicos es, más bien, que las escuelas han tendido sólo a reproducir los saberes del mercado de trabajo en sus currícula, "objetivando" y legitimando las prácticas

${ }^{25}$ Las afirmaciones de Enrique Salazar son también hechas por muchos otros periodistas a quienes entrevisté en las ciudades de Guadalajara, México y Monterrey. 
existentes, un fenómeno señalado por Raúl Fuentes desde 1991, y que explica

[...] la existencia de comunicadores en la sociedad que no han pasado por la universidad, pero cuya práctica profesional no necesariamente difiere de la de aquellos que sí han cursado la carrera y poseen un título como profesionales de la comunicación (p. 39).

\section{¿QuÉ LUGAR HA TENIDO, ENTONCES, LA ENSEÑANZA DEL PERIO- DISMO EN LA FORMACIÓN DE PERIODISTAS?}

Resultaría ocioso analizar los contenidos de los planes de estudio para intentar una respuesta a esta pregunta pues, como se ha señalado, la enseñanza del periodismo en México no ha obedecido ni a un modelo de formación de periodistas, ni a un perfil profesional deseable, en el sentido de que se mantiene la ambigüedad o polivalencia de las ofertas educativas. No obstante, es ilustrativo saber que en los planes de estudio las asignaturas relacionadas específicamente con el periodismo son muy escasas, y además es vigente uno de los reproches más fuertes que hacen los periodistas a estas escuelas: muy pocos de los maestros que enseñan periodismo lo ejercen o lo han ejercido, y algunos ni siquiera conocen una sala de redacción. ${ }^{26}$

Otra de las fuertes críticas, por parte de los periodistas, a los planes de estudio de las escuelas de comunicación, es que quieren ofrecer programas que idealmente generarían múltiples perfiles profesionales:

[Me parece realmente injusto] que se ponga en una misma carrera a gente que quiere dedicarse al periodismo y a gente que quiere dedicarse al estudio de la comunicación. [...] Poner a futuros periodistas y futuros estudiosos de la comunicación juntos, [...] para mí equivale a poner a médicos y veterinarios en una carrera. Bueno, los dos estudian tejidos y órganos, pero son carreras diferentes [...] y no sólo eso, sino gente que quiere llegar a relaciones públicas, gente que quiere dedicarse a la publicidad, como si fuera ésta una gran carrera que quisiera prepararlos a todos en sus áreas de conocimiento. A mí me parece realmente injusto. ${ }^{27}$

$\overline{{ }^{26}}$ Diversas entrevistas con periodistas en ejercicio, y Enrique Salazar (1993).

${ }^{27}$ Comunicación personal con Pascal Beltrán del Río, reportero de Proceso (ciudad de México, septiembre de 1993). 
¿Se puede hablar de un cambio en la calidad de las prácticas periodísticas por la intervención de los egresados de escuelas de periodismo?

Retomando el pretexto que dio origen a este trabajo, el de entender la exigencia social de que se "profesionalicen" las prácticas periodísticas (en el sentido de que mejoren), cabe señalar que el problema de la calidad del periodismo no se reduce, por supuesto, al de la calidad de su enseñanza en las universidades (públicas o privadas). Como práctica social que es, el periodismo es resultante de múltiples factores y actores. Hay quienes consideran que el cambio necesario en el periodismo (incrementar su calidad) debe surgir de los propios medios, no de las universidades. ${ }^{28}$ Sin embargo, hay que reconocer que se requiere repensar el o los modelos de formación universitaria, más allá de las adecuaciones técnicas y tecnológicas que exige el mercado laboral, y sabiendo que el problema de fondo es estructural y que reclamaría tanto el análisis del nivel educativo general en México (reconocidamente deficiente), como el del papel de la educación superior en el país, que, de acuerdo con los estudiosos del problema, parece estar aplazando el ingreso del cada vez mayor número de jóvenes al cada vez más restringido mercado de trabajo.

Aun aceptando todo lo anterior, ¿se puede hablar de un cambio en las prácticas periodísticas por la participación de egresados de las escuelas de periodismo y comunicación? El tema se convierte en una cuestión de opinión, en donde las apreciaciones más relevantes serían las de los formadores, los empleadores y los propios periodistas. Todos coincidirían en decir que, a pesar de todo, es mejor que los periodistas tengan escolaridad universitaria, aunque no necesariamente en comunicación o periodismo. Y los matices de las opiniones particulares serían indicativos de una respuesta a la pregunta de cuál sería el cambio en las prácticas, cuál el papel de las escuelas en la formación de periodistas.

Aunque aún son pocos, ya es mucho más frecuente encontrar periodistas que también son académicos, y a la inversa. Sus opiniones me parecen valiosas porque conocen, al menos, dos caras de un problema multidimensional.

Froylán López Narváez, del semanario Proceso, sintetiza los cambios de esta manera:

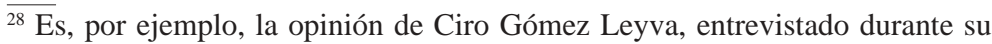
paso por Reforma (comunicación personal, febrero de 1996, ciudad de México), y de Pedro Mellado, periodista de amplia trayectoria en el estado de Jalisco. 
Y a partir de los años 75, más o menos, empiezan a aparecer "profesionales" [...] "trabajadores" sería más exacto, ya con escolaridad [...] Al salir de escuelas y con rangos o estudios académicos escolares, la actitud es otra [...] Y hay corporaciones, y libros, y conferencias y cosas así que hacen que el nuevo profesional entre como tal, como alguien que ya pertenece, no a un "oficio", sino a una "profesión". Ya es "licenciado", y ya tiene derechos [...] Entonces, ya es otra la actitud, la posición. Es decir, aunque sean ignorantes del oficio ya tiene la noción [...] hasta "estatus" [...] casi diría "clase" [...] Ya es otra la actitud, y por lo tanto otra la moral, otras las morales, aún sean incipientes ellos $[\ldots]^{29}$

\section{Jaime García Elías, de Notisistema:}

Yo creo que, cada vez más, hay un sedimento de preparación profesional, pero también creo que [...] el hecho de que estén egresando continuamente grandes cantidades de jóvenes con títulos de comunicadores, etcétera, ha saturado la profesión. De manera que encontrar periodistas o licenciados en ciencias de la comunicación, es como encontrar licenciados, o encontrar ingenieros que te los vas a encontrar tal vez trabajando de taxistas, o de cualquier otra cosa, pero no en la carrera, porque se han saturado las carreras. Entonces, es ya más la oferta que la demanda y esto debió de haberse traducido en un proceso selectivo, [en el] que prevalecieran los mejores. No necesariamente ha sido así; ha habido muchos que pudieran haber sido de los mejores, que demostraron ser muy aptos para la carrera, y que no encontraron el aliciente adecuado para perseverar en ella. Y a la inversa, mucha gente que encontró la manera de enchufarse ahí y se ha quedado. ${ }^{30}$

Hay muchas más opiniones y respuestas que con matices coinciden en lo anterior, pero elijo para concluir, porque es alentadora y crítica a la vez, la que Raúl Trejo Delarbre dio a mi pregunta expresa:

Yo creo que hay un cambio que no se advierte, que no se siente, pero que ya existe. Hoy en día por primera vez en muchos años, ya hay una generación nueva de periodistas que salieron desde hace veinte, pero sobre todo hace diez o cinco años ${ }^{31}$ de las aulas universitarias. Y que le dan una frescura nueva al periodismo. No quiero decir que pasar por la universidad dote a los estudiantes de un código ético automáticamente, hay gente

${ }^{29}$ Comunicación personal con el periodista (ciudad de México, 1993).

${ }^{30}$ Comunicación personal con Jaime García Elías, Notisistema, en Guadalajara, 1993.

${ }^{31} \mathrm{La}$ entrevista fue realizada en 1993. 
corrupta que tiene un año de haber salido de las escuelas, pero sí les da un marco de referencia distinto al de aquella persona que se ha formado sólo en la práctica profesional. Nuestros egresados de las escuelas de comunciación siguen teniendo enormes carencias, y cada vez hay mayor distanciamiento sobre todo entre quienes enseñan en las escuelas y quienes están en el campo profesional. Hay profesores de edición de diarios que nunca se han parado en una redacción, y que se pasan cada año o cada semestre repitiendo lo mismo, sin atender siquiera a las novedades técnicas que hay en todas las redacciones. Pero pese a ese hecho, el haber tenido (aunque sea con muchísmias deficiencias) una barnizadita de conocimientos generales, la posibilidad de estar en una universidad, sobre todo en el caso de las universidades públicas (y sobre todo en el caso de la UNAM), la posibilidad de haber estado en contacto con estudiantes y profesores de otras disciplinas, de haber ido al cineclub, al teatro, a pasear por el campus, todo esto da un contexto cultural, un contexto cultural novedoso por primera vez en el periodismo mexicano. Esta generación, está por hacer muchas cosas: ya está exigiendo mejores salarios, ya está compitiendo entre sí, ya hay una competencia real por los puestos de trabajo. Muy pocos que se abren en comparación con los muchos egresados de las escuelas de comunicación. Creo que hay un cambio que no se nota todavía, pero que está empezando a ocurrir. ${ }^{32}$

\section{REFERENCIAS BIBLIOGRÁFICAS}

ANDión, M. (1991) “La formación de profesionales en comunicación”, Diá.logos de la Comunicación, núm. 31, septiembre, pp. 55-63.

Baldivia, J. et al. (1981) La formación de los periodistas en América Latina (México, Chile y Costa Rica). México: CEestem/ Nueva Imagen.

Benassini, C. (2001) "Escuelas de comunicación en México: ¿realidad o imaginario social?”, Diá.logos de la Comunicación, núm. 62, Felafacs, julio, pp. 43-51.

CAletti, S. (1991) "Profesiones, historia y taxonomías: algunas discriminaciones necesarias", Diá.logos de la Comunicación, núm. 31, septiembre, pp. 25-36.

Diá.logos de la Comunicación, núm. 19 (1988), Revista de la Federación Latinoamericana de Asociaciones de Facultades de Comunicación Social (Felafacs), enero.

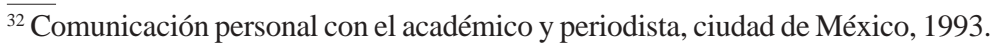


Diá.logos de la Comunicación, núm. 31 (1991), Revista de la Federación Latinoamericana de Asociaciones de Facultades de Comunicación Social (Felafacs), septiembre.

FERNÁNDEZ, F. (1997) "Escuelas de comunicación y tecnologías de la información: Binomio ¿de qué tipo?”, Diá.logos de la Comunicación, núm. 49. Lima: Felafacs, octubre, pp. 91-102.

Fuentes, R. (1988) “Escuelas de Comunicación y brechas tecnológicas en México", Diá.logos de la Comunicación, núm. 19, enero, pp. 37-45.

(1991) "Prácticas profesionales y utopía universitaria: notas para repensar el modelo de comunicador", Diá.logos de la Comunicación, núm. 31, septiembre, pp. 37-42.

(1992) "El estudio de la comunicación desde una perspectiva sociocultural en América Latina" Diá.logos de la Comunicación, núm. 32, Felafacs, marzo, pp. 16-26.

Galindo, J., y C. Luna (coords.) (1995) Campo académico de la Comunicación: hacia una reconstrucción reflexiva. México: ITESo/Consejo Nacional para la Cultura y las Artes.

GARcía, C. (2004) "La formación académica de los comunicadores multimediáticos en México”, Sala de Prensa, núm. 63, enero, año V, vol. 2. http://www.saladeprensa.org

Gargurevich, J. Gargurevich (2001) "Qué periodismo y cómo enseñarlo: una reflexión urgente”, Sala de Prensa, núm. 30, abril, año III, vol. 2. http:// www.saladeprensa.org

Godoy, A., y C. L. Herrán (1996) "La formación profesional de comunicadores sociales en América Latina”, Informe de Felafacs para las escuelas de América Latina, núm. 14, Felafacs.

LunA, C. (1991) La formación profesional de comunicadores en México: Revisión documental, tesis profesional para obtener el grado de licenciado en Ciencias de la Comunicación. Guadalajara: Instituto Tecnológico de Estudios Superiores de Occidente.

(1995) "Enseñanza profesional de la comunicación: estrategias curriculares y mediaciones metodológicas", en J. Galindo y C. Luna (eds.), Campo académico de la Comunicación: hacia una reconstrucción reflexiva. México: ITESO/Consejo Nacional para la Cultura y las Artes. 
Marques de Melo, J. (1988) "Desafíos actuales de la enseñanza de la Comunicación. Reflexiones en torno a la experiencia brasileña", Diá.logos de la Comunicación, núm. 19, enero, pp. 4-12.

(1993) "La atracción fatal de la universidad y la industria", Chasqui, núm. 44, enero, pp. 36-42.

NixOn, Raymond (1982) "Historia de las escuelas de periodismo", Chasqui, núm. 2. Quito: CIESPAL, pp. 13-19.

Prieto, D. (1988) "Notas sobre la formación del periodista", Diá.logos de la Comunicación, núm. 19, enero, pp. 30-35.

Salazar, E. (1993) "Improvisación en el arte de comunicar. Estudiantes de periodismo, candidatos al desempleo", Impacto, núm. 2272, 29 de septiembre, pp. 60-64. 